\title{
On the influence of spatial information for hyper-spectral satellite imaging characterization
}

\author{
Olga Rajadell, Pedro García-Sevilla and Filiberto Pla \\ Depto. Lenguajes y Sistemas Informáticos \\ Jaume I University, Campus Riu Sec s/n 12071 Castellón, Spain \\ (orajadel,pgarcia,pla)@1si.uji.es
}

\begin{abstract}
Land-use classification for hyper-spectral satellite images requires a previous step of pixel characterization. In the easiest case, each pixel is characterized by its spectral curve. The improvement of the spectral and spatial resolution in hyper-spectral sensors has led to very large data sets. Some researches have focused on better classifiers that can handle big amounts of data. Others have faced the problem of band selection to reduce the dimensionality of the feature space. However, thanks to the improvement in the spatial resolution of the sensors, spatial information may also provide new features for hyper-spectral satellite data. Here, an study on the influence of spectral-spatial features combined with an unsupervised band selection method is presented. The results show that it is possible to reduce very significantly the number of spectral bands required while having an adequate description of the spectral-spatial characteristics of the image for pixel classification tasks.
\end{abstract}

\section{Introduction}

Hyper-spectral images are the results of a detailed measurement of the spectra acquired by a special sensor. Currently, some sensors can easily cover a spectral resolution of $10 \mathrm{~nm}$ with a considerably high spatial resolution that can reach $1 \mathrm{~m}$ per pixel for satellite images. As a result, hyper-spectral images are composed by a very high number of correlated bands (between 200 and 500 spectral bands). Dealing with this type of images means facing a very high dimensional problem.

Since the usage of the whole hyper-spectral data set can fall into the course of dimensionality, several band selection methods have been studied in order to avoid the large amount of correlated data, while keeping the discrimination between land cover classes [1].

When the spatial resolution in hyper-spectral images was not high enough, major efforts to improve pixel classification were done focusing at the classification stage by simply using the spectral features provided by the sensors. These type of processing often used neural networks [2], decision trees [3], Bayesian estimation [4] and kernelbased methods [5] for the classification of the pixels in the images. In particular, Support Vector Machines proved to obtain good performances in this task [6].

Because of the increase in the spatial resolution, spectral-spatial analysis has been lately an issue of high interest for the improvement of hyper-spectral imaging characterization [7] which is widely used for tasks like land-cover classification and segmentation of remote sensing images. Some basic spatial features have been used like the mean value of a $N \times N$ window around a pixel, the standard deviation of the values 
in this window, and the combination of the mean and standard deviation for a series of window sizes [6]. On the other hand, textural analysis has been widely discussed to study the spatial relationships in an image. This sort of features could be applied over hyper-spectral images in order to have a better description of the spectral-spatial properties. There exists a huge variety of methods [8]: co-occurrence matrices, wavelet analysis, Gabor filtering, Local Binary Patterns, etc.

It is likely that improving the characterization of the image may help to reduce even more the amount of spectral bands required for the classification task. To pursue this goal, we have chosen two different spectral-spatial characterization methods. In first place, simple statistics (mean and standard deviation) of the $N \times N$ neighbors around a pixel will be considered for each spectral band. Later, a Gabor filter bank will be used to obtain features to describe the pixel in each band. Spectral-spatial feature extraction will be presented in Section 2. The hyper-spectral database used in our experiments is described in Section 3. The spectral-spatial methods proposed provide an improvement over the spectral classification as will be shown in Section 4. Finally, we draw out conclusions in Section 5.

\section{Integration of spatial information in imaging characterization methods}

Pixel characterization aims at obtaining one feature vector for each pixel to be used in a pixel classification task in a multidimensional space. When only spectral data is used, the feature vector for every pixel is defined as the spectral curve provided by the sensor. The target of a spectral-spatial characterization method is to calculate a feature vector using the spectral data given and this can whether replace the spectral feature vector or being combined with it.

Let $I^{i}(x, y)$ be the $i^{t h}$ band of an image containing $B$ bands. When the spectral curve is used as the feature vector for each pixel in the image this vector is simply composed of the values provided by the sensor, that is:

$$
\psi_{x, y}=\left\{I^{i}(x, y)\right\}_{i=1}^{B}
$$

\subsection{Basic spatial characterization}

Spectral-spatial analysis of the image is based on a series of values extracted from spatial operations involving its neighbor pixels (spatial features) [9]. Frequently the two statistics used are the mean and the standard deviation of the neighborhood. This is a very simple method to include spatial information obtaining only 2 features per pixel [6].

Let $M_{n}^{i}(x, y)$ be the window $n \times n$ centered in the pixel $(x, y)$ of the spectral band $i$. Then, the feature vector for this pixel is defined by:

$$
\phi_{x, y}=\left\{\operatorname{mean}\left(M_{n}^{i}(x, y)\right), \text { standard_deviation }\left(M_{n}^{i}(x, y)\right)\right\}_{i=1}^{B}
$$


It is also possible to concatenate the features calculated from several window sizes (i.e. $n=3,5,7,9)$ increasing the size of the vector $\phi$ depending on the number of windows used. This provides a multi-scale or multi-resolution description of the image.

\subsection{Feature extraction based on Gabor filters}

Several features have been suggested in the literature for the description of texture information [8]. In this paper Gabor filtering will be used because, in general, they have provided the best results in different sort of texture characterization experiments [10] [11]. In this case, features are obtained by filtering the input image with a set of filters. The set of outputs obtained for each pixel in the image forms its feature vector. Here, the filter bank is defined to be a set of two-dimensional Gabor filters. Each Gabor filter is characterized by a preferred orientation and a preferred spatial frequency (scale). The filter acts as a local band-pass filter with optimal joint localization properties in the spatial domain and the frequency domain [12].

Gabor filters consist essentially of sine and cosine functions modulated by a Gaussian envelope. They can be defined by equation (3) where $m$ is the index for the scale, $n$ for the orientation and $u_{m}$ is the central frequency of the scale [12].

$$
f_{m n}(x, y)=\frac{1}{2 \pi \sigma_{m}^{2}} \exp \left\{-\frac{x^{2}+y^{2}}{2 \sigma_{m}^{2}}\right\} \times \cos \left(2 \pi\left(u_{m} x \cos \theta_{n}+u_{m} y \sin \theta_{n}\right)\right)
$$

Notice that set the condition $f_{m n}(0,0)=0$ dismisses completely the effect of the measurements themselves and making the analysis independent from the pixel spectral values themselves.

Note that Gabor filters will be used in this case as a multi-dimensional extension of the technique designed for mono-channel images. In this way, multi-spectral images will be simply decomposed into separated channels and the same feature extraction process will be performed over each channel as shows equation (4).

$$
h_{m n}^{i}(x, y)=I^{i}(x, y) * f_{m n}(x, y)
$$

These responses are used to construct the final feature vector for each pixel.

$$
\Upsilon_{x, y}=\left\{h_{m n}^{i}(x, y)\right\}_{\forall i, m, n}
$$

\section{Hyper-spectral data set}

To pursue the experimental campaign a widely used hyper-spectral database has been used, 92AV3C, known as AVIRIS. Figure 1 show a color composition, its corresponding ground-truth and the classes in it.

Hyper-spectral image data 92AV3C was provided by the Airborne Visible Infrared Imaging Spectrometer (AVIRIS) and acquired over the Indian Pine Test Site in Northwestern Indiana in 1992. From the 220 bands that composed the image, 20 are usually ignored because of the noise (the ones that cover the region of water absorption or with low SNR [7]). The image has a spatial dimension of $145 \times 145$ pixels. Spatial resolution 


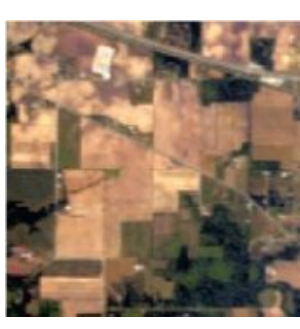

(a)

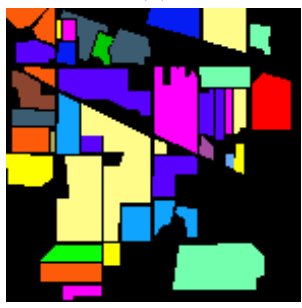

(b)

\begin{tabular}{|c|c|c|}
\hline class & color & No. of samples \\
\hline \hline Stone-steel towers & & 95 \\
\hline Hay-windrowed & & 489 \\
\hline Corn-min till & & 834 \\
\hline Soybeans-no till & & 968 \\
\hline Alfalfa & & 54 \\
\hline Soybeans-clean till & & 614 \\
\hline Grass/pasture & & 497 \\
\hline Woods & & 1294 \\
\hline Bldg-Grass-Tree-Drives & & 380 \\
\hline Grass/pasture-mowed & & 26 \\
\hline Corn & & 234 \\
\hline Oats & & 20 \\
\hline Corn-no till & & 1434 \\
\hline Soybeans-min till & & 2468 \\
\hline Grass/trees & & 747 \\
\hline Wheat & & 212 \\
\hline
\end{tabular}

(c)

Fig. 1. Hyper-spectral image AVIRIS (92AV3C). a)Color composition. b)Ground-truth. c)Target classes contained.

is $20 \mathrm{~m}$ per pixel. Fig. 1 shows the sixteen available classes, ranging from 20 to 2468 pixels in size. In it, three different growing states of soya can be found, together with other three different growing states of corn, woods, pasture and trees are the larger classes in terms of number of samples (pixels). Due to the small size of the rest of classes they are frequently dismissed in literature. In this paper, we will perform experiments using both 16 and 9 classes.

\section{Spectral/spatial classification results}

As it has been pointed out, remote sensing has to deal with high dimensional feature vector where features are highly correlated. Consequently, band selection methods are frequently used. In our case, a band selection method presented by Martinez et al. in [1] has been used. Let $D$ be a number of spectral bands such as $D \leq B$, where $B$ is the total number of bands included in the database. This method provides the best set of $D$ bands in term of uncorrelated information. It is based on a clustering approach that joins groups of bands depending on their mutual information. Once a partition of $D$ groups is available, a representative band from each group is selected.

\subsection{Classification task}

In Figures 2 and 3 a global view of the classification results using different spectralspatial characterization methods can be found. The classification rates using only spectral information has also been included to be considered as a baseline reference. These results show the overall accuracy for four different sizes of windows to extract spatial 
information of the pixels $(3 \times 3,5 \times 5,7 \times 7,9 \times 9)$, the combinations of these spatial features which is just a concatenation of all of them, and the Gabor textural features using two scales and four orientations. Every characterization method has been tested with the corresponding set of bands provided by the band selection algorithm from 1 to 15. Also the task with all bands in the dataset has been performed and can be observed in Table 1.

In these experiments, the pixels that form the whole image were divided into twenty non overlapping sets, keeping the a priori probability of each class. Therefore, no redundancies are introduced and each set is a representative set of the original image. The same sets of pixels are used in all experiments. Ten classification attempts were carried out with the k-nearest neighbor classifier with $k=3$ and the mean of the error rates of these attempts was taken as the final performance of the classifier for this experiment. Each classification attempt uses one of these sets for training and another set for testing. Each set is never used twice, so the attempts are totally independent.

Experiments using all 16 available classes are shown in Figure 2. As an alternative, experiments excluding the classes with a reduced number of samples have also been carried out using the same criterion as in [6]. Their results are presented in Figure 3. Better results, as expected, were got in this case. Small classes represent small structures in the image that are hard to recognize since their size is not enough to be capture by spatial features. Furthermore, some neighborhoods may be too big that several spatial structures could be considered at a time.

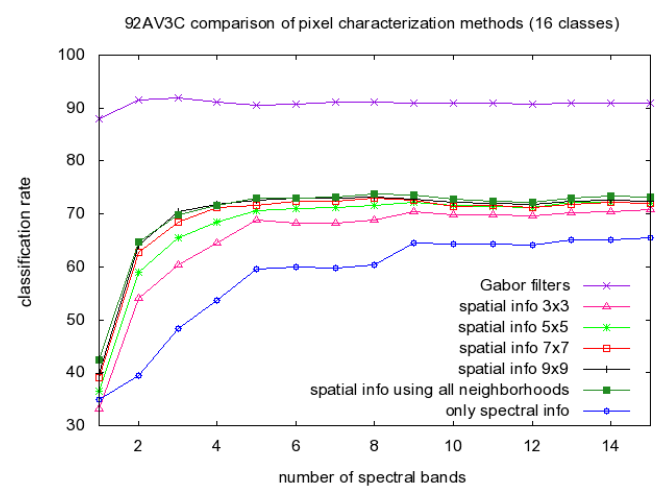

Fig. 2. Pixel classification rates for the $92 \mathrm{AV} 3 \mathrm{C}$ database using all 16 classes. The number of spectral bands selected varies from 1 to 15 .

Significant differences were obtained between spectral-spatial features and only spectral features even if the basic spatial features were used. Regarding these last sort of features, observe also that the larger the neighborhood used, the better classification results were obtained. Also, the concatenation of features obtained using different window sizes did not improve the results provided by using only the largest window. This means that, in this case, the spatial characterization is more reliable when we describe pixels by a fairly stable neighborhood. Furthermore, Gabor textural features 


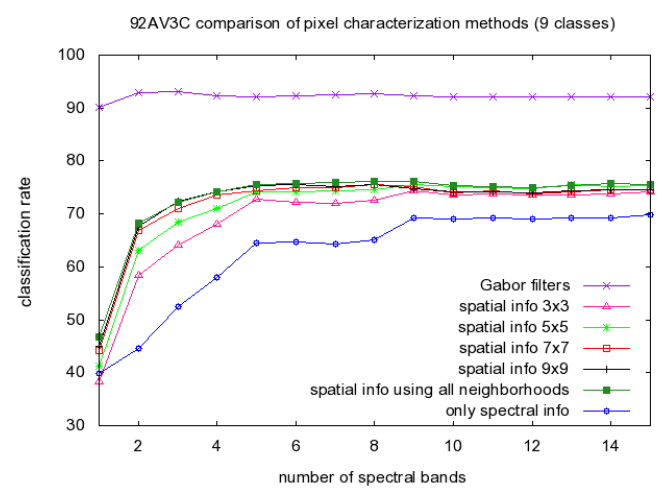

Fig. 3. Pixel classification rates for $92 \mathrm{AV} 3 \mathrm{C}$ database using only the main 9 classes. The number of spectral bands selected varies from 1 to 15 .

outperformed all other methods very significantly. This points out that detailed spatial information is really discriminative for land use classification in this sort of images.

The differences between the characterization methods are not only due to the final classification rates obtained. Note also, that the number of spectral bands required to reach the stable behavior (where more spectral bands do not improve the classification results) is quite different. While spectral features require more than 12 bands, basic spatial features reach the stable zone with only 6-8 bands, while Gabor textural features required only $2-3$ spectral bands.

In Table 1 the results obtained for several numbers of spectral bands can be compared with those obtain when using all 200 bands. Notice that, no matter the set of features used, no improvement is obtained by increasing the incoming data although the size of the problem is considerably increased.

\begin{tabular}{c|c|c|c|c|} 
& \multicolumn{4}{|c|}{ Characterization methods } \\
\hline $\begin{array}{c}\text { \# of } \\
\text { spectral bands }\end{array}$ & $\begin{array}{c}\text { Spectral } \\
\text { information }\end{array}$ & $\begin{array}{c}\text { Spatial } \\
\text { window } 9 \times 9\end{array}$ & $\begin{array}{c}\text { Spatial } \\
\text { All windows }\end{array}$ & $\begin{array}{c}\text { Gabor } \\
\text { features }\end{array}$ \\
\hline \hline 1 & 34.964 & 39.916 & 42.367 & 88.049 \\
\hline 3 & 48.361 & 70.451 & 69.851 & 91.885 \\
\hline 5 & 59.652 & 72.612 & 72.939 & 90.553 \\
\hline 7 & 59.765 & 72.957 & 73.212 & 91.036 \\
\hline 9 & 64.534 & 72.879 & 73.635 & 90.977 \\
\hline 200 & 52.849 & 73.521 & 73.633 & 90.456 \\
\hline
\end{tabular}

Table 1. Accuracy for the 16 classes classification experiments of the $92 \mathrm{AV} 3 \mathrm{C}$ dataset using different features. Results from the first sets of bands have been included together with the results obtained using the complete database (200 spectral bands).

\subsection{Segmentation results}

Since the problem we are tackling involve land-use pixel classification, the percentages of correct may not be enough to appreciate the goodness of the results. Pixel classifica- 
tion experiments assign a class label to each pixel in the test set. If we represent these labels in the position of their corresponding pixels we will obtain a segmentation map of the processed image. In Figure 4 this representation of the results is shown where misclassified pixels (errors) are represented in white color, while the rest of pixels are represented by their own class color as presented in the ground-truth shown in Figure 1. The results shown correspond to classification experiments where only one set of pixels was used for training (5\% of the pixels in the image) and the other 19 sets of pixels were used for testing, using 10 spectral bands. Only the results for three characterization methods are shown. On the left, the results using the basic spatial features extracted from all different window sizes $(3 \times 3,5 \times 5,7 \times 7,9 \times 9)$ are shown. Results using Gabor features are shown in the center of the figure. The results obtained using only spectral information are presented on the right.

Observe how the errors are distributed over the different classes. Spectral features (on the right) suffer from salt and pepper classification noise since the error is all over the areas an is not localized. However, when using Gabor textural features, the errors are located mainly in small areas and at the borders of the classes were the spatial features are mixing information from the heterogenous background. We could say that the areas recognized using these features are more homogeneous. In the case of the basic spatial features, the errors are distributed in a similar way to the ones obtained using Gabor features but the results are worse in this case, so the misclassified pixels extent deeper inside the classes.

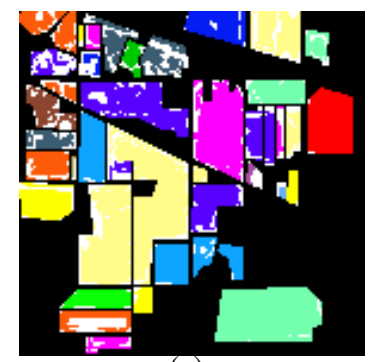

(a)

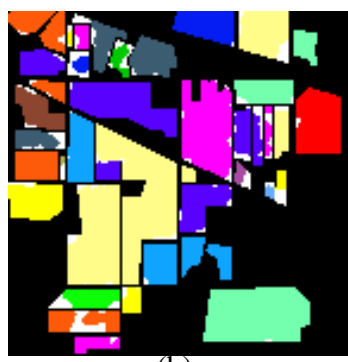

(b)

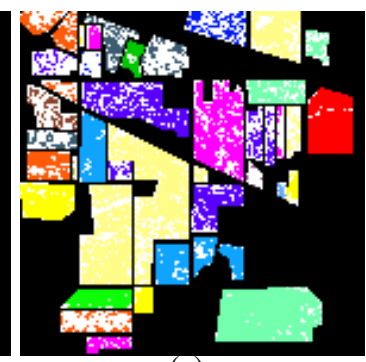

(c)

Fig. 4. Pixel classification results using 5\% of the pixels for training for the 16 classes of the 92AV3C database, using 10 spectral bands. (a) Basic spatial features for all window sizes considered (b) Gabor textural features (c) Spectral features.

\section{Conclusions}

An experimental campaign over the $92 \mathrm{AV} 3 \mathrm{C}$ dataset has been performed using several spectral-spatial characterization methods. Among them, the basic spatial features using simple statistics derived from a neighborhood and a Gabor textural features for a filter bank with two scales and four orientations have been used. Both basic and Gabor features outperform the naive spectral classification pointing out that taking advantage of the spatial resolution in the image is highly recommended for pixel classification tasks. 
Besides, Gabor textural features have provided very good classification results using a basic K-nearest neighbor classifier. Spectral features never provided results close to the ones obtained using spatial information even when all two hundred spectral features were considered. In the segmentation experiments, spatial features have also proven their good performance providing quite homogenous regions and keeping the classification errors near the boundaries of the classes due to the influence of the heterogenous background. Furthermore, the good classification results obtained using spatial features required a minor number of spectral bands. Therefore, the use of spatial information can reduce the number of spectral bands required for pixel classification tasks and, at the same time, improve the rates of pixel classification.

\section{Acknowledgments}

This work has been partly supported by grant FPI PREDOC/2007/20 from Fundació Caixa Castelló-Bancaixa and projects CSD2007-00018 (Consolider Ingenio 2010) and AYA2008-05965-C04-04 from the Spanish Ministry of Science and Innovation.

\section{References}

1. Martínez-Usó, A., Pla, F., García-Sevilla, P.: Clustering-based hyperspectral band selection using information measures. IEEE Trans. on Geoscience \& Remote Sensing 45 (2007) 41584171

2. Yang, H., Meer, F., Bakker, W., Tan, Z.: A back-propagation neural network for mineralogical mapping from aviris data. International Journal of Remote Sensing 20 (1999) 97-110

3. Zhou, H., Mao, Z., Wang, C.: Classification of coastal areas by airbone hyperspectral image. In: Proceedings of SPIE. (2005) 471-476

4. Chen, C., Ho, P.: Statistical pattern recognition in remote sensing. Pattern Recognition $4 \mathbf{1}$ (2008) 2731-2741

5. Camps-Valls, G., Bruzzone, L.: Kernel-based methods for hyperspectral image classification. IEEE Trans. on Geoscience \& Remote Sensing 43 (2005) 1351-1362

6. et al., A.P.: Recent advances in techniques for hyperspectral image processing. Remote sensing of environment 113 (2009) 110-122

7. Landgrebe, D.A.: Signal Theory Methods in Multispectral Remote Sensing. Hoboken, NJ: Wiley (2003)

8. Petrou, M., García-Sevilla, P.: Image Processing: Dealing with Texture. John-Wiley and Sons (2006)

9. Jimenez, L., Landgrebe, D.: Hyperspectral data analysis and supervised feature reduction via projection pursuit. IEEE Trans. on Geoscience and Remote Sensing 37(6) (November 1999) $2653-2667$

10. Manjunath, B., Ma, W.: Texture features for browsing and retrieval of image data. IEEE Trans. on Pattern Analysis and Machine Intelligence 18(8) (August 1996) 837 -842

11. Rajadell, O., García-Sevilla, P., Pla, F.: Filter banks for hyperspectral pixel classification of satellite images. In: CIARP 2009, Lecture Notes in Computer Science. Volume 5856., Springer (2009) 1039-1046

12. Fogel, I., Sagi, D.: Gabor filters as texture discriminator. Biological Cybernetics 61 (1989) 103-113 